Rapid Reviews COVID-19

\title{
Review 1: "Rapid 'mix and read' assay for scalable detection of SARS-CoV-2 antibodies in patient plasma"
}

Xi Chen ${ }^{1}$, Simin Xia ${ }^{2}$

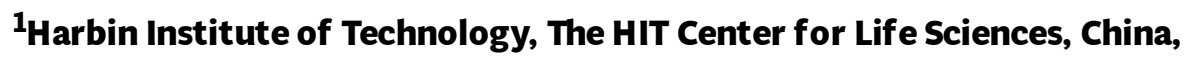

${ }^{2}$ MVM, The HIT Center for Life Sciences

Published on: Nov 15, 2020

DOI: $10.1162 / 2 \mathrm{e} 3983 f 5.6 \mathrm{c} 7 \mathrm{ffb} 2 \mathrm{e}$

License: Creative Commons Attribution 4.0 International License (CC-BY 4.0). 


\section{$\underline{\text { RR:C19 Evidence Scale rating by reviewer: }}$}

- Reliable. The main study claims are generally justified by its methods and data. The results and conclusions are likely to be similar to the hypothetical ideal study. There are some minor caveats or limitations, but they would/do not change the major claims of the study. The study provides sufficient strength of evidence on its own that its main claims should be considered actionable, with some room for future revision.

$* * * * * * * * * * * * * * * * * * * * * * * * * * * * * * * * * * * * * * * *$

\section{REVIEW}

General comments: In this study, Yue etc. introduced a rapid "mix and read" assay for scalable detection of SARS-CoV-2 antibodies in patient plasma. This assay is based on the detection of a ternary complex between antigen and serum antibodies using a time resolved FRET readout (TR-FRET). A fluorophore donor, Terbium (emission at 490 $\mathrm{nm}$ ) is situated at the SARS-CoV-2 spike protein antigen while the fluorophore accepter, BODIPY (emission at $520 \mathrm{~nm}$ ) is located at the anti-hsIgG antibody. Once the anti-S-protein antibody is presented in CoV2 + patent's sample, Terbium labeled SARSCoV-2 spike protein and BODIPY labeled anti-hsIgG will simultaneously bind to anti-Sprotein, thus bringing the Terbium and BODIPY FRET pair to proximity. In this case, the fluorescence emission ratio between 520/480 nm will increase, indicating that the serological testing result is positive. The authors found that Terbium conjugation to the antigen results in optimal performance when used with serum/plasma samples. In addition, it seems that Degree of Labelling (DOL) of $\sim 3.8$ results in no detectable epitope masking with optimal signal. Finally, the authors also show that this method could be extended to detect other antigens, such as SARS-CoV-2 N-protein, suggesting the versatility of this approach. In all, this work seems to a reliable study that provides a valuable tool for simple and scalable detection of SARS-CoV-2 serological samples. More specific comments are given below which include advantages and disadvantages of this new detection method, and possible ways to further strengthen this study.

Advantage: Easy implementation seems to be the key advantage of this detection approach. Compared to other nucleic acid-based approaches, as well as other serological detection methods (e.g. ELISA), the reported detection method is very simple-just add $<1 \mu$ l of plasma sample to the detection cocktail and then allow microplate reader to read fluorescence; this easy implementation provides scalability of this approach. 
Disadvantages: There are also few limitations associated with this method. i) Lack of amplification may be the major drawback of this approach. Although the authors show that this approach could still provide some sensitive detection results; yet readers may still believe other detections approaches, e.g. RT-PCR, RT-RPA, ELISA etc. that includes amplification step(s) could be more sensitive. ii) The reliability of this approach may also be a concern to some extent. In some of the detection results, false negatives and false positives are presented. This may raise concerns of the reliability of this detection results.

Potential ways of optimization: i) In the abstract, it may be better to briefly mention the basic principle of the detection method in a greater detail. ii) As for detecting proximity via FRET, the authors chose Terbium as the donor and BODIPY as the acceptor fluorophore. Since FRET signal and background is highly dependent on the FRET pair chosen, the authors could briefly note why they chose this FRET pair; ii) since false positive and false negative results are presented, did the authors re-validate the serological samples they used via PCR? 